\title{
ON THE DISTANCE TO SINGULARITY VIA LOW RANK PERTURBATIONS
}

\author{
Christian Mehl, Volker Mehrmann and Michą Wojtylak
}

Abstract. For regular matrix pencils the distance in norm to the nearest singular pencil under low rank perturbation is studied. Characterizations of this distance are derived via the Weyl function of the perturbation. Special attention is paid to the Hermitian pencil case. Estimates for the distance of a given pencil to the set of singular pencils are obtained.

Mathematics subject classification (2010): 15A18, 15A22, 47A75, 65F15, 65F22.

Keywords and phrases: Low rank perturbation, singular pencils, distance to singularity, Weyl function.

\section{REFERENCES}

[1] H. Attouch, J. Bolte, P. Redont, And A. Soubeyran, Proximal alternating minimization and projection methods for nonconvex problems: an approach based on the Kurdyka-tojasiewicz inequality Math. Oper. Res., 35: 438-457, 2010.

[2] K. E. Brenan, S. L. Campbell, and L. R. Petzold, Numerical Solution of Initial-Value Problems in Differential Algebraic Equations, SIAM Publications, Philadelphia, PA, 2nd edition, 1996.

[3] R. Byers, C. He, AND V. Mehrmann, Where is the nearest non-regular pencil, Linear Algebra Appl., 285: 81-105, 1998.

[4] F. DE TERÁn AND F. DOPICO, Low rank perturbation of regular matrix polynomials, Linear Algebra Appl., 430: 579-586, 2009.

[5] F. DE TERÁN AND F. DOPICO, First order spectral perturbation theory of square singular matrix polynomials, Linear Algebra Appl., 432: 892-910, 2010.

[6] F. DE TERÁN, F. DOPICO, AND J. MORO, First order spectral perturbation theory of square singular matrix pencils, Linear Algebra Appl., 429: 548-576, 2008.

[7] E. Eich-Soellner And C. FüHrer, Numerical Methods in Multibody Systems, B. G. Teubner Stuttgart, 1998.

[8] F. R. Gantmacher, Theory of Matrices, Chelsea, New York, 1959.

[9] I. Gohberg, P. Lancaster, And L. Rodman, Indefinite Linear Algebra and Applications, Birkhäuser, Basel, 2005.

[10] G. H. Golub And C. F. Van Loan, Matrix Computations, Johns Hopkins University Press, Baltimore, 3rd edition, 1996.

[11] N. J. Higham, D. S. Mackey, N. MACKey, AND F. Tisseur, Symmetric linearizations for matrix polynomials, SIAM J. Matrix Anal. Appl., 29 (1): 143-159, 2006.

[12] T. Kato, Perturbation Theory for Linear Operators, Springer Verlag, New York, N.Y., 1966.

[13] S. Krantz and H. Parks, A Primer of Real Analytic Functions, Birkhäuser, Boston, MA, 2nd edition, 2002.

[14] P. Kunkel and V. Mehrmann, Differential-Algebraic Equations. Analysis and Numerical Solution, EMS Publishing House, Zürich, Switzerland, 2006.

[15] R. Lamour, R. März, and C. Tischendorf, Differential-Algebraic Equations: A Projector Based Analysis, Differential-Algebraic Equations Forum, Springer Verlag, 2013.

[16] D. Mackey, N. Mackey, C. Mehl, And V. Mehrmann, Structured polynomial eigenvalue problems: Good vibrations from good linearizations, SIAM J. Matrix Anal. Appl., 28 (4): 1029-1051, 2006. 
[17] The MathWorks, Inc., Natick, MA, Simscape, 2013., http://www.mathworks.de/products/ simscape/.

[18] The MathWorks, Inc., Natick, MA, Simulink, 2013., http://www.mathworks.de/products/ simulink/.

[19] MATLAB, Version r2013b, The MathWorks, inc., 24 Prime Park Way, Natick, MA 01760-1500, USA, 2013.

[20] Modelica Association, Modelica standard library 3.2.1., https://www.modelica.org/.

[21] A. C. M. RAN AND M. WOJTYLAK, Eigenvalues of rank one perturbations of unstructured matrices, Linear Algebra Appl., 437: 589-600, 2012.

[22] R. RIAZA, Differential-algebraic systems. Analytical aspects and circuit applications, World Scientific Publishing Co. Pte. Ltd., Hackensack, NJ., 2008.

[23] S. S AVCHENKO, On the Perron roots of principal submatrices of co-order one of irreducible nonnnegative matrices, Linear Algebra Appl., 361: 257-277, 2003.

[24] S. SAVCHENKO, Typical changes in spectral properties under perturbations by a rank-one operator, Mat. Zametki, 74: 590-602, 2003., (Russian). Translation in Mathematical Notes, 74: 557-568, 2003.

[25] S. SAVCHENKo, On the change in the spectral properties of a matrix under a perturbation of a sufficiently low rank, Funktsional. Anal. i Prilozhen, 38: 85-88, 2004., (Russian). Translation in Funct. Anal. Appl. 38: 69-71, 2004.

[26] R. THOMPSON, The characteristic polynomial of a principal subpencil of a Hermitian matrix pencil, Linear Algebra Appl., 14: 135-177, 1976. 